\title{
LA EXPLOTACIÓN DEL ALFAR DE LA TROYA EN EL TIEMPO: CASUALIDAD O MEMORIA (DEPARTAMENTO TINOGASTA, CATAMARCA, ARGENTINA)
}

\author{
THE EXPLOITATION OF CERAMIC RAW MATERIAL OF LA TROYA \\ ALONG TIME: CHANCE OR MEMORY \\ (TINOGASTA DEPARTMENT, CATAMARCA, ARGENTINA)
}

\author{
Norma Ratto*, Martín Orgaz** y Rita Plá***
}

\begin{abstract}
La oralidad es el mecanismo principal de transmisión de conocimientos y creencias en las sociedades tradicionales donde la memoria cumple un papel destacado. Diferentes investigaciones llevadas a cabo en la región de los Andes demostraron que diversas prácticas culturales son mantenidas a través de la memoria social. En este trabajo se presentan y discuten los resultados del análisis por activación neutrónica realizados sobre tiestos y depósitos arcillosos, los que establecen que el alfar de La Troya, localizado en el Bolsón de Fiambalá, fue explotado para la manufactura de artefactos cerámicos que abastecieron a las instalaciones puneñas y otras localizadas en la amplia cuenca del Abaucán (Departamento Tinogasta, Catamarca, Argentina) desde las sociedades Formativas hasta el arribo de los Inkas a la región. Con base en esos resultados pensamos que la explotación y apropiación en el tiempo de un mismo alfar no es casualidad sino que obedece a la memoria que reproduce la práctica social en el tiempo. Asimismo, evaluamos los datos arqueológicos y analíticos obtenidos para comprender el proceso propio de desarrollo cultural del valle de Abaucán y su relación con el área puneña meridional.
\end{abstract}

Palabras claves: explotación de arcillas, período Formativo, Inka, memoria social, activación neutrónica.

Oral tradition is an important mechanism for transmission of knowledge and belief in prehistoric societies where memory has an outstanding role. Different investigations carried out in the Andean region have demonstrated that diverse cultural practices are maintained through social memory. We present and discusses the results from a neutron activation analysis of potsherds and clay deposits which demonstrate that clay raw material from La Troya, located at the Bolsón de Fiambalá, was used for the manufacture of ceramic artifacts. These artifacts supplied Puna instalations as well as others at the Abaucán basin (Tinogasta Department, Catamarca, Argentina) from the Formative epoch to the Inka arrival in the region. Based on these results, we think that exploitation and appropriation of the same clay for a long period of time is not fortuitous, but rather is the consequence of a long process of social memory reproduction.

Key words: exploitation of clay, Formative period, Inka, social memory, neutron activation analysis.

Nuestra área de estudio se localiza en el oeste del Departamento Tinogasta de la provincia de Catamarca (Argentina). La estructura del registro arqueológico regional permite sostener que la región puneña del curso superior del valle de Chaschuil funcionó como un corredor para la circulación de bienes, energía e información donde los loci de mayores densidades artefactuales se comportaron como nodos de una red, interconectando instalaciones y diferentes espacios utilizados por sociedades agropastoriles (Formativas) y estatales (Inka). El resultado es la conformación de paisajes arqueológicos superpuestos que materializan la estructura sociopolítica y espacial de las sociedades que ocuparon los pisos de altura (Ratto 2000, 2003; Ratto y Orgaz 2000; Ratto et al. 2002 a y b, entre otros). Por su parte, los análisis por activación neutrónica realizados sobre tiestos y depósitos arcillosos y de inclusiones minerales indican que las sociedades Formativas e Inka explotaron el mismo alfar -fuente de aprovisionamiento de materia prima cerámica- para la manufactura de ceramios, con los que abastecieron las instalaciones puneñas y otras localizadas en el valle mesoter-

\footnotetext{
* Museo Etnográfico Juan B. Ambrosetti (FFyL-UBA) y Escuela de Arqueología (UNCa). Moreno 350, Buenos Aires (1092), Argentina.

** Escuela de Arqueología, Universidad de Catamarca. Av. Belgrano 300, S. F. del V. de Catamarca (4700), Argentina.

**** Técnicas Analíticas Nucleares. Comisión Nacional de Energía Atómica, Av. del Libertador 8250, Buenos Aires (1429), Argentina.
} 
mal. El área de aprovisionamiento se localiza en el Bolsón de Fiambalá, valle de Abaucán (Dpto. Tinogasta, Catamarca), específicamente en el área de La Troya que también es el lugar de emplazamiento del sitio arqueológico Batungasta. Aproximadamente $100 \mathrm{~km}$ lineales es la distancia entre este sector del valle mesotérmico y la región puneña.

El gran desafío consiste en interpretar los datos analíticos suministrados por las ciencias físico-químicas en el contexto histórico-arqueológico de las sociedades del pasado, especialmente con la dinámica interna de cada valle o territorio. Surgen algunas preguntas: (a) ¿Qué comportamiento social está siendo representado por los resultados analíticos obtenidos por activación neutrónica?; (b) ¿Cuál es el mecanismo de transmisión para la reproducción parcial o total de determinadas prácticas sociales en sociedades con diferentes organizaciones sociopolíticas?, y (c) ¿Cuál es el alcance e integración de los resultados obtenidos dentro del desarrollo sociocultural regional?

Asumimos que toda sociedad conforma conjuntos organizados cuyos elementos se encuentran en continua interacción y se relacionan con el medio ambiente circundante. Dollfus (1991) manifiesta que los flujos de información que reciben los sistemas sociales pueden ser percibidos como dos grandes memorias: la de la naturaleza y la del tiempo de los hombres, encontrándose ambas siempre presentes y en constante interacción. Algunos elementos de la memoria de la naturaleza pueden ser utilizados por las sociedades dado que conocen su valor y poseen los conocimientos producidos por el saber técnico para concebirlos como recursos naturales que se convierten en bienes cuando son transformados por el trabajo. La memoria del tiempo de los hombres se alimenta de la historia, conllevando fines, metas, valores, significados, símbolos e intencionalidades que se transmiten a través de la lengua, de la escritura, del diseño plástico, de las prácticas sociales, es decir, diferentes acciones que fundamentan la vida en común, el conocimiento de las técnicas, el saber, en definitiva, todo lo que se transmite por la formación y la herencia. Sin memoria no hay contrato, convención, sociedad, identidad individual y colectiva, y saber (Candau 2002). Puede decirse que vivimos de herencias materiales e inmateriales dando como resultado que ninguna sociedad puede ignorar su pasado. De esta manera, el espacio geográfico es explorado, explotado, transitado, habitado y manejado por los grupos sociales. La consecuencia es que a medida que se reproduce la sociedad ésta reproduce su espacio condicionando indefectiblemente las relaciones. Por consiguiente, el espacio geográfico, concebido de esta manera, contiene dos dimensiones intrínsecamente relacionadas: una física y la otra social. La consecuencia es la conformación de diferentes paisajes culturales que son producto de la memoria de la naturaleza y del tiempo de los hombres, determinando que algunos lugares, rasgos geográficos, prácticas sociales u objetos porten un compendio diverso de saber cultural y social por conllevar información y valorización que es trasmitido y compartido a través del ritual.

La memoria resulta un aspecto importante en la vida cotidiana, principalmente en las comunidades tradicionales. En los Andes, diferentes rasgos de la sociedad perviven en el tiempo y son explicados a través de la memoria. Diversos aspectos de la cosmogonía andina fueron indagados a través del estudio de los textiles por contener un lenguaje propio difícil de descifrar por los conquistadores (Bubbá 1997; Desrosiers 1997), constituyendo además un soporte material importante para los procesos de memorización y de transmisión. Los mitos estatales pintados en qeros coloniales buscaban conservar en la memoria de los miembros de los linajes reales la historia que justificaba su gobierno, constituyendo una forma de mantener vivo el mito histórico (Flores Ochoa 1990). Por otra parte, el recuerdo de rutas y sus espacios son pensados o recordados a través de un sistema mnemotécnico donde las ch 'allas o las visitas rituales тиуи recuerdan el paisaje, empleándose para ello mapas mentales (Harris 1997). En este mismo sentido Arnold (1998) considera que la construcción de una casa en la comunidad de Qaqachaka (Bolivia) constituye una práctica social con un fuerte trasfondo mnemotécnico, a modo de sendas de la memoria, que le permite a los miembros del grupo recordar periódicamente las memorias colectivas de sus ancestros y muertos. Sin embargo, la memoria no es estática ya que es enriquecida y recreada continuamente por la creación humana. En este proceso continuo una parte pervive, otra se pierde o se transforma, quedando como gran desafío para la historia y la arqueología el poder revivirlas. Recientemente, Hastorf (2003) evaluó el papel de la memoria social como un componente importante para explicar la formación de la comunidad en el 
período Formativo medio de Chiripa (Bolivia). Evidentemente es un problema por demás complejo que nos plantea diferentes preguntas que no son dominio de una única disciplina. Es por ello que el desafío sólo puede encararse desde diferentes perspectivas, interrelacionando arqueología, historia y ciencias físico-químicas para comprender cómo el comportamiento humano queda reflejado en los artefactos y sus distribuciones (Arnold et al. 1999; Bouysse-Cassagne 1997).

Con base en lo expuesto, el objetivo del trabajo es contextualizar los resultados analíticos dentro del proceso de desarrollo sociocultural regional para discutir:

1. La explotación y apropiación en el tiempo del alfar de La Troya para determinar si obedece a la casualidad o si nos encontramos ante la representación de una práctica social que a través de la memoria se materializa en estilos tecnológicos (sensu Stark 1999) o representaciones sociales (sensu Lemonier 1992), es decir, en la forma en que las cosas son siempre hechas por los artesanos. En dichos conceptos quedan representados la suma de los procesos técnicos: las materias primas, las fuentes de energía, las herramientas y los conocimientos específicos, constituyendo estas elecciones las bases del conocimiento de una tradición manufacturera que es pasada de una generación a la próxima. Nos interesa discutir qué elecciones perviven, cuáles se transforma o se pierden como una forma de pensar la multitemporalidad social, es decir, la simultaneidad de diferentes historias engranadas por la memoria social dentro de un mismo territorio.

2. Cuál es la relevancia y el alcance que tiene la práctica de la reutilización de un alfar en el tiempo dentro del desarrollo regional de los valles de Abaucán y Chaschuil.

\section{Materiales, Métodos y Características de la Muestra}

\section{Conformación de la muestra}

La muestra procesada por activación neutrónica está conformada por fragmentos cerámicos y depósitos arcillosos e inclusiones minerales procedentes de sitios arqueológicos y lugares de extracción de materias primas cerámicas localizados en distintas ecozonas del área puneña del valle de Chaschuil y del mesotermal de Abaucán (Dpto. Tinogasta, Catamarca). De acuerdo con la Periodización Cultural del Noroeste Argentino catamarqueño, las sociedades prehispánicas involucradas en la muestra cerámica se clasifican en distintas etapas del desarrollo agropastoril hasta el control estatal, adjudicándoles bases económicas y organizaciones sociopolíticas con distintos grados de complejidad, encontrándose además distanciadas por cientos de años unas de otras (Sempé 1976, 1977; entre otros).

Los sitios arqueológicos representan diferentes momentos dentro del desarrollo cultural regional, existiendo en algunos casos reocupación de espacios en el tiempo (Ratto y Orgaz 2000; Ratto 2003). El material cerámico asociado fue clasificado en unidades cerámicas definidas para el noroeste argentino en función de sus características estilísticas y tecnológicas, privilegiando su alta inclusividad y baja ambigüedad para garantizar la obtención de resultados similares por parte de diferentes investigadores.

a) 108 fragmentos cerámicos se adscriben a la etapa agropastoril de los Períodos Temprano y Medio (Formativo) procedentes de sitios arqueológicos localizados en puna y valle -Tabla 1. Estos se caracterizan por presentar baja carga de antiplásticos, predominando el cuarzo y aún en menor proporción mica, feldespatos y fragmentos de rocas. El tamaño de grano oscila entre muy fino a fino, estimándose que puede tratarse de un componente natural del depósito explotado. La cocción es predominantemente reductora y en menor proporción oxidante (Feely 2003; Ratto et al. 2002b).

b) 194 fragmentos cerámicos se adscriben a la etapa agropastoril del Período Tardío (pre Inka) e Inka procedentes de sitios arqueológicos de puna y valle -Tabla 1. Estos se caracterizan por presentar una carga de antiplásticos que oscila entre el 20 al $25 \%$, predominando el cuarzo, la calcita, fragmentos de rocas, mica e inclusiones arcillas en proporciones que varían levemente entre el material inka y pre Inka. El tamaño de antiplástico varía entre medio y fino, con excepción del material pre Inka, donde se observa mayor rango de variabilidad, mientras que la cocción es oxidante en todos los casos (Ratto et al. 2002a). 
Tabla 1. Conjunto cerámico analizado por activación neutrónica en función del sitio arqueológico de procedencia, área de localización y adscripción cultural.

Pottery sample analyzed by neutronic activation in relation to site function, localization and cultural epoch.

\begin{tabular}{|c|c|c|c|c|c|c|}
\hline \multirow{2}{*}{ Sitio Arqueológico } & \multirow{2}{*}{ Área } & \multicolumn{4}{|c|}{ Adscripción de la muestra cerámica } & \multirow{2}{*}{$\begin{array}{c}\text { Total } \\
\text { muestra } \\
\text { cerámic }\end{array}$} \\
\hline & & $\begin{array}{l}\text { Período } \\
\text { Temprano }\end{array}$ & $\begin{array}{l}\text { Período } \\
\text { Medio }\end{array}$ & $\begin{array}{c}\text { Período } \\
\text { Tardío }\end{array}$ & Inca & \\
\hline San Francisco (4.000 msm) & Puna & - & - & - & 74 & 74 \\
\hline El Corral $(4.000 \mathrm{msm}$ & Puna & 15 & 9 & - & - & 24 \\
\hline Las Coladas (4.200 msm) & Puna & - & - & - & 13 & 13 \\
\hline Ojo de Las Lozas (4.000 msm) & Puna & 7 & 1 & 1 & 1 & 10 \\
\hline Tambería (4.000 msm) & Puna & - & - & - & 4 & 4 \\
\hline Ranchillos 1 (2.425 msm) & Valle & - & - & 2 & 1 & 3 \\
\hline Batungasta (1.480 msm) & Valle & 14 & 8 & 66 & 10 & 98 \\
\hline Costa de Reyes (1.465 msm) & Valle & - & - & 4 & 4 & 8 \\
\hline Laguna Salada (3.980 msm) & Puna & 23 & 4 & - & - & 27 \\
\hline Las Grutas $(3.980 \mathrm{msm})$ & Puna & - & - & - & 3 & 3 \\
\hline Las Cuevas (4.000 msm) & Puna & 3 & - & - & - & 3 \\
\hline Palo Blanco (1.980 msm) & Valle & 14 & - & - & - & 14 \\
\hline Tatón 1 (2.700msm) & Valle & 10 & - & - & - & 10 \\
\hline $\begin{array}{l}\text { Campo de Grabados de } \\
\text { Guanchincito }(1.700 \mathrm{msm})\end{array}$ & Valle & - & - & 3 & - & 3 \\
\hline $\begin{array}{l}\text { Cementerio Finca Justo Pereyra } \\
(1.980 \mathrm{msm})\end{array}$ & Valle & - & - & 4 & - & 4 \\
\hline $\begin{array}{l}\text { Hornos periferia de Batungasta } \\
(1.490 \text { a } 1470 \mathrm{msm})\end{array}$ & Valle & - & - & 4 & - & 4 \\
\hline \multirow[t]{2}{*}{ Totales } & & 86 & 22 & 84 & 110 & \multirow[t]{2}{*}{302} \\
\hline & & $\begin{array}{l}108 \mathrm{fr} \\
- \text { For }\end{array}$ & $\begin{array}{l}\text { ntos } \\
0-\end{array}$ & $\begin{array}{r}194 \\
- \text { Pre }\end{array}$ & $\begin{array}{l}\text { ntos } \\
\text { Inca- }\end{array}$ & \\
\hline
\end{tabular}

El resto del conjunto está compuesto por 48 muestras procedentes de depósitos arcillosos localizados en: (a) el valle mesotermal de Abaucán, abarcando el curso inferior $(1.500 \mathrm{msm})$ y superior (3.000 msm) del río La Troya, el río El Puesto (1.480 msm), el río Guanchín (desde la cota de 1.500 a $3.000 \mathrm{msm}$ ) y el río Colorado ( $1.465 \mathrm{msm})$. Todos los depósitos muestreados pertenecen a la formación Calchaquense (Terciario).

Los depósitos arcillosos procedentes del río $\mathrm{La}$ Troya, tanto en su curso inferior $(\mathrm{n}=18)$ como superior $(n=2)$, constituyen arcillas secundarias, meteorizadas y transportadas por agentes fluviales en un ambiente netamente sedimentario. En general, la región presenta alta disponibilidad de materias primas cerámicas, con excelentes propiedades de plasticidad y trabajabilidad para la manufactura cerámica. Los resultados del análisis por difracción de rayos $\mathrm{X}$ de las muestras procedentes del curso inferior indican que son de buena calidad. Presentan altos porcentajes de arcilla (17\%-45\%), con importantes concentraciones de minerales arcillosos: esmectita-montmorillonita e illita; porcentajes relativamente bajos de feldespatos $(10 \%-25 \%)$ y calcita $(2 \%-5 \%)$, y altos de cuarzo (24-45\%). Estas inclusiones minerales constituyen un antiplástico natural de la formación del depósito. También se realizaron muestreos en el curso superior del río La Troya donde los depósitos presentan mayor fracción arenosa y menor plasticidad 
con relación a los del curso inferior. Por otra parte, los depósitos de los ríos El Puesto $(\mathrm{n}=5)$ y Colorado $(n=3)$ ofrecen características macroscópicas similares a los del área de La Troya -curso inferior-, encontrándose a una distancia de 8 y $50 \mathrm{~km}$, respectivamente. Por último, las muestras provenientes de Guanchín $(n=19)$ tienen características diferenciales, ya que las que provienen de cotas superiores a los $2.000 \mathrm{msm}$ presentan mayor fracción arenosa y menor plasticidad que las obtenidas en cota inferior. Estas últimas se asemejan en su textura a las procedentes del curso inferior del río La Troya, distante $25 \mathrm{~km}$ aproximadamente (De La Fuente 2002). Además, se recuperaron tres muestras de arcillas utilizadas hoy día por las ceramistas locales, las que provienen de la localidad de Fiambalá y Medanitos.

\section{Procedimientos para la Preparación de las Muestras y Tratamiento Numérico de los Datos}

El empleo de la técnica analítica de análisis por activación neutrónica (AAN) permite la caracterización de elementos que tienen una marcada diferenciación geoquímica durante el proceso de cristalización de las rocas ígneas cuya meteorización dio origen a las arcillas que luego fueron utilizadas para la manufactura de vasijas. Por lo tanto, conforman una herramienta útil para la diferenciación de los depósitos arcillosos. Las pastas cerámicas son un sistema complejo constituido básicamente por dos componentes: (a) minerales arcillosos y (b) inclusiones no-plásticas o temperantes, que pueden estar presentes en la composición original de la arcilla o adicionarse durante el proceso de manufactura. Esto determina que la interpretación de los resultados químicos no sea lineal ni trivial, dado que las relaciones originales de concentración entre varios elementos en la arcilla pueden ser alteradas por la adición de temperante. Esta es la razón de la importancia de conocer la composición mineralógica de la muestra cerámica a ser analizada por AAN (Bishop 1980; Bishop et al. 1982; Bishop y Neff 1989; Neff 1993, 1998; entre otros). En general, las concentraciones de elementos traza tienden a ser menos afectados por la inclusión de temperantes como arena, calcita, fragmentos de rocas o vegetales, que por materiales volcánicos o inclusiones arcillosas (Bishop et al. 1982).
Para asegurar la homogeneidad de la muestra se siguió el siguiente procedimiento: (i) se utilizó un fragmento de tiesto de $2 \mathrm{~cm}^{2}$ ( 2 a 3 gr), aproximadamente; (ii) se limpió la superficie raspándola con una lima de carburo de tungsteno, descartando el polvo resultante; (iii) se molió el fragmento en un mortero de ágata y el polvo obtenido se secó en estufa a $105^{\circ} \mathrm{C}$ por 24 horas; (iv) luego se dejó enfriar en un desecador, y (v) se conservó la muestra en un envase de vidrio hasta su análisis. Las muestras fueron preparadas y analizadas en el laboratorio del Grupo de Técnicas Analíticas Nucleares del Centro Atómico Ezeiza de la Comisión Nacional de Energía Atómica (Argentina). Se irradiaron masas de muestra de $100 \mathrm{mg}$, aproximadamente, envasadas en ampollas de cuarzo y colocadas en cápsulas de aluminio junto con materiales de referencia adecuados. Las irradiaciones se realizaron en el reactor RA-3 (flujo térmico $3.10^{13} \mathrm{~cm}^{-2} \cdot \mathrm{s}^{-1}$; $4,5 \mathrm{Mw}$ ) por 5 horas. Se hicieron dos mediciones con 7 y 30 días de decaimiento y se determinaron 22 elementos: $\mathrm{As}, \mathrm{Ba}, \mathrm{Ce}, \mathrm{Co}, \mathrm{Cr}, \mathrm{Cs}, \mathrm{Eu}, \mathrm{Fe}, \mathrm{Gd}$, Hf, La, Lu, Nd, Rb, Sb, Sc, Sm, Ta, Tb, Th, U y $\mathrm{Yb}$. Las mediciones se realizaron usando detectores Ortec de Ge HP (30\% de eficiencia y resolución 1,8 keV para el pico de 1332,5 keV del Co60 ), acoplados a un módulo Buffer multicanal Ortec 919 y empleando el programa GammaVision para la adquisición de los datos. Para cuantificar se empleó el material de referencia certificado NIST SRM 1633b Coal Fly Ash y las concentraciones se calcularon usando un programa desarrollado en el laboratorio del grupo analítico.

Los resultados del análisis por duplicado del $10 \%$ de las muestras mostraron un buen acuerdo. La reproducibilidad del método analítico fue probada analizando los materiales de referencia estándar NIST 2709 San Joaquín Soil, NIST 699 Brick Clay y USGS AGV. Los resultados fueron comparados con los producidos por los laboratorios de AAN del Instituto de Pesquisas Energéticas e Nucleares de Brasil y de la Comisión Chilena de Energía Nuclear, obteniéndose un acuerdo con el $95 \%$ de confianza para $\mathrm{Ce}, \mathrm{Co}, \mathrm{Cr}, \mathrm{Cs}, \mathrm{Eu}$, $\mathrm{Fe}, \mathrm{Hf}, \mathrm{La}, \mathrm{Sc}, \mathrm{Sm}$, Th y Yb. Además, cinco muestras cerámicas fueron también analizadas por los laboratorios mencionados y la Universidad de $\mathrm{Pa}$ via (Italia). Los resultados obtenidos mostraron un buen acuerdo entre laboratorios.

Se realizó un análisis numérico multivariado por Componentes Principales (CP) -Programa Es- 
tatística 5.5-, realizándose la transformación logarítmica de los datos, matriz de covarianza y rotación Varimax. Para el análisis estadístico se seleccionaron sólo aquellos elementos que reunían las siguientes condiciones: (i) sus mediciones presentaban un error menor al 10\%; (ii) fueron medidos en todas las muestras, y (iii) se contaba con información suficiente sobre su comportamiento a través de la literatura especializada (Bishop 1980; Bishop et al. 1982; Bishop y Neff 1989; Neff 1992; entre otros). Con base en lo expuesto, fueron seleccionados los elementos $\mathrm{Ce}, \mathrm{Eu}, \mathrm{La}, \mathrm{Lu}, \mathrm{Sm}, \mathrm{Yb}$ y $\mathrm{Tb}$ (Tierras Raras-Grupo III), Th (Actínido-Grupo III), Ba, Cs, Rb (Alcalinos-Grupo I) y Sc, Hf, $\mathrm{Co}, \mathrm{Cr}$, Ta y Fe (Elementos de Transición). El Sb y $\mathrm{U}$ presentan las condiciones impuestas por los dos primeros criterios de selección pero no cumplen con el tercero. El Ta y Tb sólo se incluyeron en el análisis estadístico de los datos analíticos de los depósitos arcillosos, dado que se registró la ausencia de sus valores para el $15 \%$ de la muestra de fragmentos cerámicos.

Las muestras provenientes de los depósitos arcillosos fueron analizadas sin tratamiento previo de elaboración de briquetas. Esto determinó que se agregara un 10\% a los valores de concentración obtenidos para compararlas con los datos de la matriz multielemental de los fragmentos cerámicos (Ronald Bishop comunicación personal 2000).

\section{Resultados Analíticos y su Contexto Arqueológico}

La base empírica conformada por: (a) la composición multielemental de fragmentos cerámicos y depósitos arcillosos; (b) las características tecnológicas de la muestra, y (c) la evidencia de 27 estructuras de combustión registradas en la periferia del sitio arqueológico Batungasta, conformaron el soporte para la discusión de las relaciones regionales para modelar las características de la producción y distribución de los bienes cerámicos entre la puna de Chaschuil y el valle mesotermal de Abaucán (Plá y Ratto 2000; Ratto et al. 2002a; Ratto y Orgaz 2002; entre otros). Al estado actual del conocimiento puede decirse que:

a) El análisis estadístico multivariado de las muestras de los depósitos arcillosos del valle de Abaucán $(n=51)$ define dos componentes que explican el 81,403\% de la varianza total.
Se analizaron aquellos elementos que son diagnósticos para determinar diferencias entre depósitos cercanos. El primer componente se define por la carga aportada por las TTRR, con excepción del Eu y el Th; mientras que en el segundo por el Ta, Co, Cr y Eu. La elipse de confiabilidad de las muestras procedentes del río La Troya engloba a muestras localizadas dentro de un radio de aproximadamente 25 $\mathrm{km}$ que se ubican dentro de la cota altitudinal de $1.500 \mathrm{msm}$. La mayoría de las muestras que quedan fuera de la elipse de confiabilidad se localizan en pisos altitudinales más altos (Figura 1).

b) Los tiestos clasificados dentro de diferentes estilos cerámicos, correspondientes a distintas etapas del desarrollo cultural del Noroeste Argentino, presentan muy alta similitud en sus perfiles composicionales multielementales. Esta afirmación es válida tanto para los tiestos procedentes de sitios arqueológicos del área puneña de Chaschuil como del valle de Abaucán, con la excepción de algunos tiestos Ciénaga y Aguada (Formativo) y pre Inkas. La superposición de casos en el espacio factorial bivariado está reflejando la presencia de un único perfil composicional multielemental, interpretándose que los tiestos localizados dentro de la nube o grupo principal fueron manufacturados con la misma fuente de aprovisionamiento de materia prima cerámica, diferenciándose de los que se posicionan fuera de aquella. De acuerdo con el 'criterio de abundancia' (Bishop et al. 1982) se infiere que el valle fue el área para la producción cerámica por ofrecer condiciones adecuadas que se materializan en la presencia de: (a) depósitos de arcilla e inclusiones minerales; (b) combustible vegetal; (c) características climáticas óptimas para sostener una producción anual, y (d) la evidencia de 27 estructuras de combustión arqueológicas para la manufactura cerámica que se localizan en la periferia del sitio Batungasta (Ratto et al. 2002 a). La reclamación de este espacio se materializa hoy día por el testimonio arquitectónico y artefactual que da cuenta de la presencia Inka e Hispano Indígena. Sin embargo, la abundancia de material con estilos cerámicos propios del Formativo (Ciénaga, Saujil, Aguada, entre otros) permite pensar que el área fue 

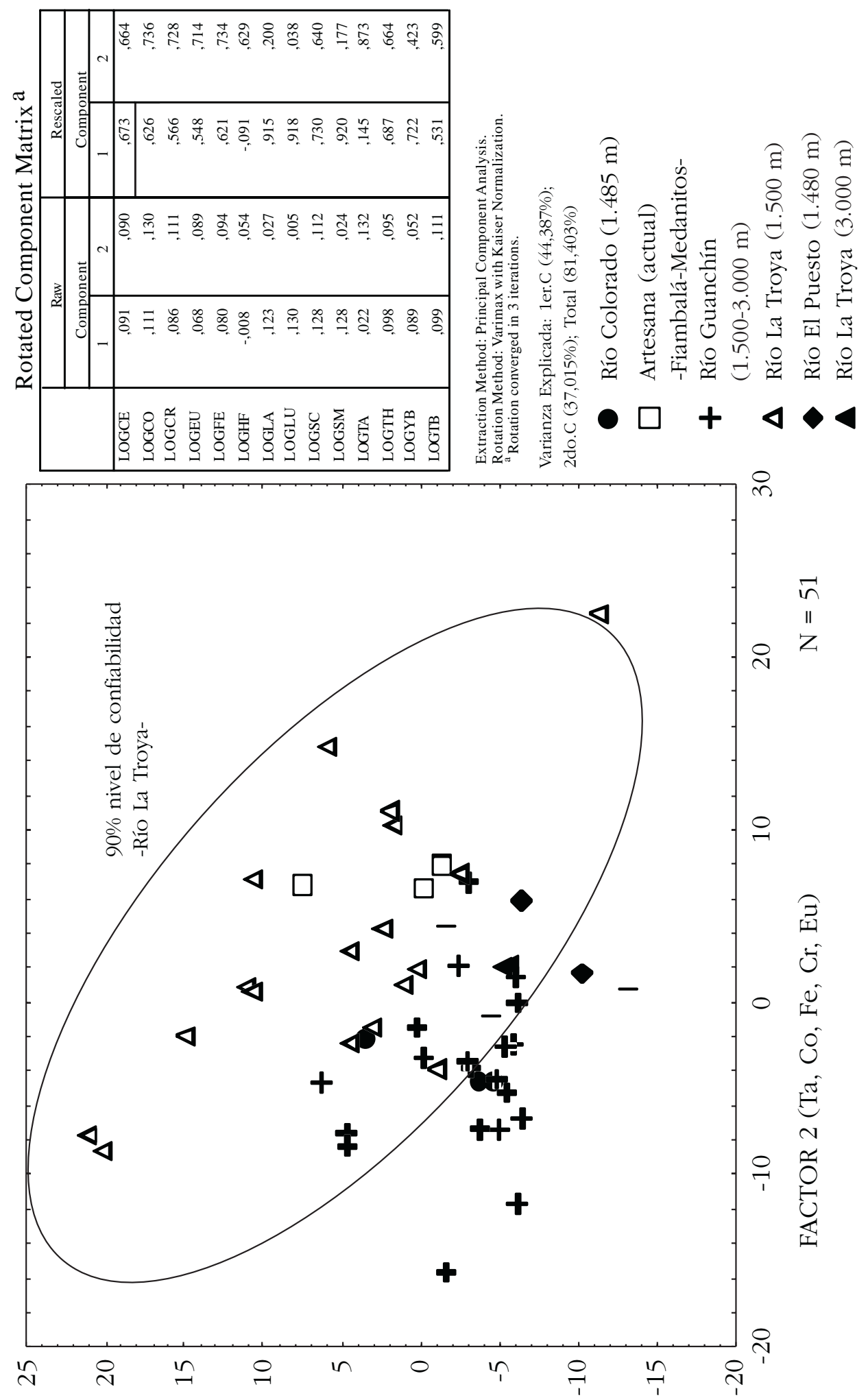

(วว 'บ.L 'qX 'วS 'nT 'eT 'US) I บOLJVH 
explotada por estos grupos para el aprovisionamiento de materias primas, manufactura y circulación de bienes cerámicos. $\mathrm{Al}$ respecto, los tiestos provenientes de la periferia de $\mathrm{Ba}$ tungasta, asignados a la etapa Formativa, pero sin calibración temporal absoluta, guardan un perfil composicional multielemental similar con aquellos procedentes de sitios Formativos emplazados en la puna (Figura 2), los que fueron posteriormente reclamados por la ocupación incaica en la región. Las dataciones por TL posicionan temporalmente entre el 550 al 1.160 años de la era a los fragmentos cerámicos recuperados de estos sitios puneños, conociéndose que fueron manufacturados con arcillas procedentes del área mesotérmica de La Troya (Ratto et al. 2002b). Los fechados se alejan de los rangos temporales fijados por el esquema de periodización tradicional del noroeste argentino, constituyendo un dato más en la ardua tarea de redefinir y calibrar una secuencia cultural "general" dentro de la dinámica particular de cada valle. Para los fines de este trabajo es importante conocer que alrededor del año 1.200 de la era se manufacturaron vasijas con materias primas del alfar de La Troya que guardan características tecnológicas y estilísticas que permiten adscribirlas a la etapa Formativa.

c) Finalmente, el tratamiento numérico multivariado realizado con los datos analíticos de la misma muestra cerámica $(\mathrm{n}=298)$ más aquellos provenientes de los depósitos muestreados $(n=51)$, establece que el perfil químico del depósito de La Troya es el que guarda mayor similitud con el de los tiestos cerámicos Formativos, pre Inka e Inka, tanto de valle como de Puna (Figura 3).

La contextualización de los resultados analíticos con la evidencia arqueológica recuperada en las estructuras de combustión documentadas en la periferia de Batungasta permitió corroborar la hipótesis de que Batungasta funcionó como un centro de producción y distribución de bienes cerámicos durante tiempos incaicos para abastecer a las instalaciones puneñas de Chaschuil, como así también a otras localizadas en el valle de Abaucán (Ratto et al. 2002a). Sin embargo, aún no hemos recuperado material cerámico perteneciente al Formativo dentro de las estructuras de combustión excavadas hasta la fecha. Lo concreto es que una misma área de aprovisionamiento de materias pri- mas cerámicas se utiliza a lo largo del tiempo dentro de contextos sociohistóricos diferentes.

\section{Discusión}

Los análisis texturales y mineralógicos realizados sobre las muestras de los depósitos arcillosos localizados en la cota de $1.500 \mathrm{msm}$ indican que presentan calidades similares, pudiéndose inferir que todos serían potencialmente utilizables. El resultado de la investigación en el sitio Batungasta permite decir que la instalación fue emplazada en un área con recursos arcillosos, combustibles e hídricos y con características climáticas adecuadas para la manufactura cerámica; estas condiciones más el hallazgo de las estructuras de combustión y los resultados analíticos permiten calificarlo como un centro manufacturero de bienes cerámicos para la época incaica (Ratto et al. 2002a). Puede pensarse que la localización del sitio se debe a la proximidad de los recursos críticos necesarios para la producción cerámica. Sin embargo, queda el interrogante de por qué la reutilización de un alfar en el tiempo cuya calidad no es superior a otros cercanos localizados en cotas altitudinales similares. $\mathrm{Al}$ respecto, es interesante destacar que las artesanas actuales locales consideran a las arcillas de $\mathrm{La}$ Troya como 'de mala calidad'.

Dado que nuestros estudios se realizan en los Andes meridionales donde las poblaciones mantienen una relación de reciprocidad con el paisaje, concibiéndolo como animado, es que pensamos que la explotación y reutilización del alfar de La Troya en el tiempo se debe a aspectos estructurales interrelacionados de la sociedad. Por una parte, en este contexto resulta pertinente la definición dada por Sallnow (1989) de 'Economía moral andina' donde la explotación de recursos mineros, incluida la arcilla, sólo es comprendida dentro de su contexto cultural. Los estudios etnográficos indican que las comunidades andinas consideran que las arcillas pertenecen a un espíritu local, debiéndose realizar ofrendas para su extracción tal como queda documentado:

“... In many communities clay is also conceived of as belonging to a local spirit, and the extraction can only be justifies through the making of an appropriate offering. In Pumpri an offering is made to the clay mines on the first of August... These offerings are given by the male potters" ( $\mathrm{Si}-$ llar 1994:107). 

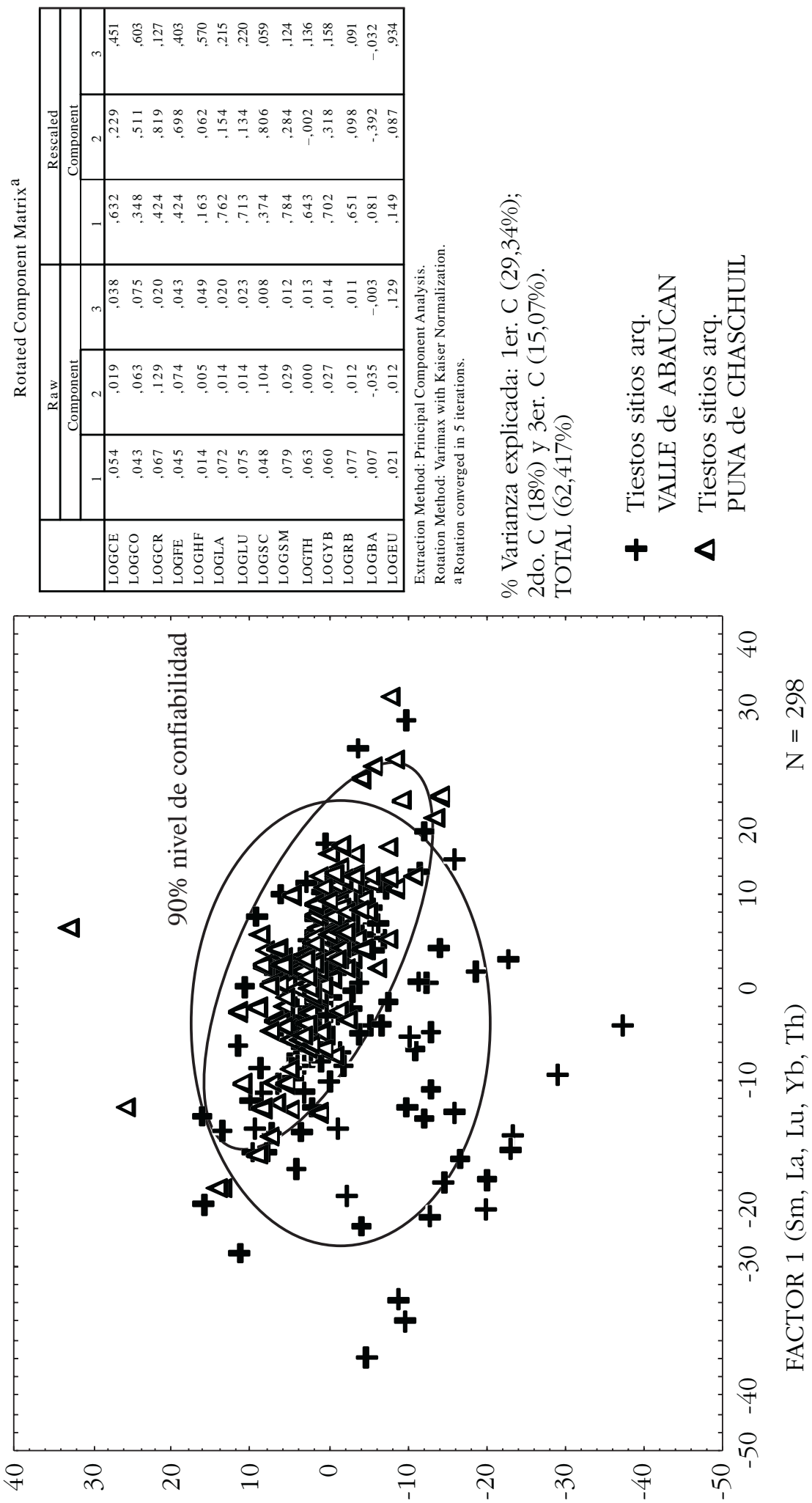

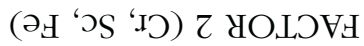




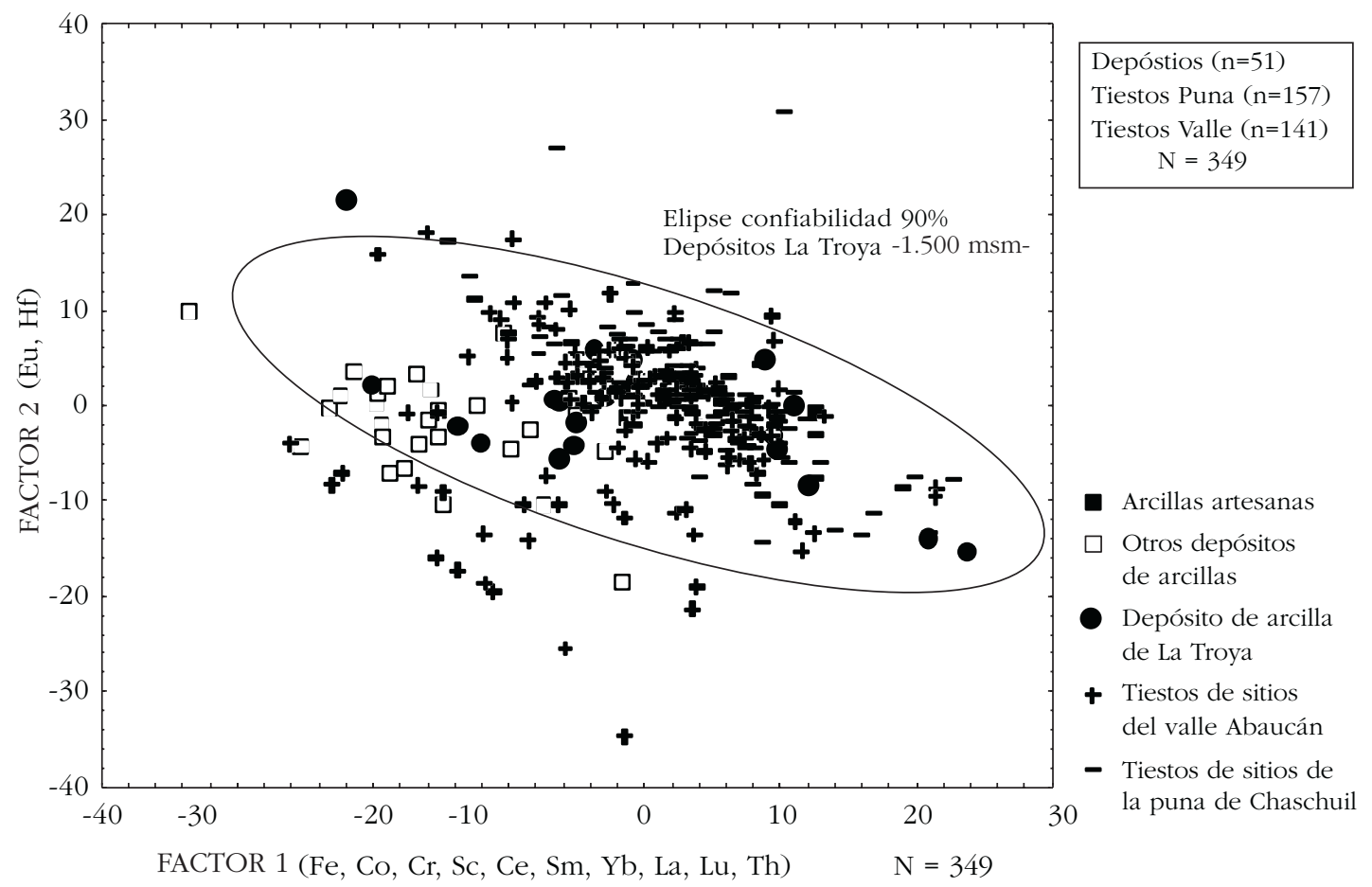

Figura 3. Disposición en el espacio factorial de la composición multielemental de los tiestos provenientes de puna y valle, el alfar de La Troya y otros depósitos de bancos de arcilla de la región.

Spatial distribution of the multicomponent composition of vessels from puna and valley the quarry of La Troya and other clay bank deposits.

La etnohistoria ofrece una imagen similar a través de Hernández de Príncipe (1923), quien en el siglo XVII documenta prácticas rituales y capacochas en pueblos de olleros con la finalidad de obtener buena arcilla para la manufactura cerámica y favorecer la continuidad del recurso (Zuidema 1989a y b). Por lo tanto, el valor asignado a estos minerales y el costo de su explotación no pueden explicarse a través del binomio "costo-beneficio', ya que su extracción es mediatizada por una serie de prácticas sociales y ofrendas rituales con la finalidad de pedir permiso para el acceso al recurso porque pertenece a los Apus y Wamanis.

La tecnología en los Andes es un producto social que no tiene como único fin dar respuestas a las necesidades inmediatas. Pensamos que la producción de cerámica es una práctica que se realiza no sólo para satisfacer requisitos materiales primarios. Las actividades donde se registra su presencia son amplias y en muchos casos están dirigidas para mantener buenas relaciones con los muertos, las deidades y/o relacionadas con la construcción de relaciones sociales en el mundo terrenal. Un estudio etnoarqueológico, realizado en diferentes localidades de los Andes, demostró que la producción de alfarería cumple un papel importante en la reproducción social del grupo, vinculándose con el mundo sobrenatural a través de su uso en el consumo de chicha y la manufactura de miniaturas. De esta manera, dentro de la economía andina, la producción de vasijas no sólo es necesaria para cumplir con las actividades primarias, sino que excede ampliamente este plano cuando constituyen objetos que se utilizan en la mediación para asegurar la reproducción de las relaciones sociales, tanto a nivel doméstico como regional (Sillar 1994).

Los mitos, los relatos históricos, los ritos y las creencias colectivas constituyen una suerte de cápsula histórica donde los pueblos concentran su memoria social sin la cual no hay identidad. La formación social de la memoria nos remite a una pregunta clave: su modo de transmisión. De acuerdo con Connerton (1989) consideramos que las imágenes y el conocimiento recuperado del pasado son transmitidos por los grupos sociales a través de rituales en donde se comparten memorias a 
través de prácticas, definiéndolas como ceremonias conmemorativas que no pueden ser comprendidas fuera de su contexto cultural.

En nuestro caso la memoria se reproduce dentro de contextos sociohistóricos específicos que todavía no podemos precisar, pero el perfil químico multielemental de la muestra cerámica analizada indica que los bienes fueron manufacturados con materias primas arcillosas procedentes de la misma área geográfica a lo largo del tiempo, siendo coincidente con el perfil químico multielemental del depósito de La Troya. En otras palabras, de los resultados analíticos inferimos la continuidad de una práctica en el tiempo que se encuentra inmersa dentro de contextos sociohistóricos diferentes, pero donde prevalece la transmisión de 'cómo se hacen las cosas', a manera de una trama que une diferentes urdimbres para conformar un proceso histórico enraizado en la memoria andina.

Resulta sugestivo el resultado analítico que establece el empleo de un mismo banco de arcilla a través del tiempo. Esto nos induce a buscar respuestas para esta elección tecnológica en los aspectos estructurales de las sociedades que ocuparon y explotaron ese espacio, ya que están condicionando o dirigiendo la selección de cierto alfar y no de otro. Creemos que este suceso no es un gesto menor si consideramos a la tecnología como un acto social, dado que la materia prima le pertenece a las deidades y los objetos a ser manufacturados constituyen instrumentos que en los Andes funcionan como intermediarios en la reproducción social de una comunidad. Nuestra interpretación es que la pervivencia en el tiempo constituye una práctica social que es reproducida por la memoria, la que es transmitida y legitimada a través de la realización de alguna modalidad de ceremonias conmemorativas que aún no estamos en condiciones de precisar.

Lo concreto es la reproducción de prácticas que incorporan el uso de los mismos espacios del valle y de la puna a lo largo de la historia regional, es como si con la llegada del Inka no se produjera una ruptura porque se antepone la memoria de los lugares y los espacios. A la luz de estos resultados proponemos que las poblaciones agropastoriles del Abaucán y el Chaschuil con modalidades propias de economías Formativas perduraron en el tiempo más allá de los rangos temporales fijados por el modelo de periodización propuesto para la región por Sempé (1976). Estas poblaciones interactuaron con otras, pero nos aventuramos a plantear que esas relaciones recién se intensificaron en momentos de la ocupación incaica, posiblemente también relacionado con el movimiento de pueblos que caracterizó esta conquista. La continuidad de la práctica social se refuerza sobre la base de considerar la interacción entre distintos contextos sociohistóricos que representan entidades culturales alejadas en el tiempo para los modelos de periodización en vigencia. De esta manera, el uso compartido de un alfar puede constituir una estrategia de negociación de las poblaciones locales del valle con los Inkas, ya que para explotarlas es necesario contar con el permiso de sus verdaderos dueños, los espíritus locales. Además, permite que los Inkas se incorporen a la estructura social de la comunidad local, apoyándose en la memoria social para legitimar sus derechos, solucionar conflictos y/o construir alianzas.

Los resultados de los análisis por activación neutrónica nos han desafiado para realizar una lectura teórica alternativa para analizar el problema de la reocupación y reutilización de espacios extractivos y productivos de una región en el tiempo. Esto fue posible porque abordamos el estudio de la dinámica cultural regional a través de una escala temporal amplia que integra diferentes organizaciones sociopolíticas que se sucedieron y/o interrelacionaron a lo largo del tiempo. Este acercamiento al problema puso de manifiesto qué parte de las elecciones técnicas realizadas por los Inkas constituyeron una práctica desarrollada desde larga data por otros contextos sociohistóricos, asignándole particularidades propias a la ocupación de la región, dada la diferencia de las estrategias implementadas en otros territorios ocupados por el Estado, por ejemplo para el valle de Mantaro (D’Altroy y Bishop 1990). Sólo el avance de las investigaciones dará mayor resolución a muchos de los temas aquí discutidos.

Agradecimientos: Los análisis por activación neutrónica se realizaron en el marco del Proyecto ARG 9393 financiado por la Internacional Atomic Energy Agency. 


\section{Referencias Citadas}

Arnold, D.

1998 La casa de adobes y piedras del Inka: género, memoria y cosmos en Qaqachaka. En Hacia un Orden Andino de las Cosas, editado por D. Arnold, A. D. Jiménez y J. de Dios Yapita, pp. 31-108. HISBOL-ILCA, La Paz.

Arnold, D., H. Neff, R. Bishop y M. Glascoc

1999 Testing interpretative assumptions of neutron activation analysis. En Material Meaning. Critical Approaches to the Interpretation of Material Culture, editado por Elizabeth Chilton, pp. 61-84. The University of Utah Press, Salt Lake.

Bishop, R.

1980 Aspects of ceramic compositional modeling. En Models and Methods in Regional Exchange, editado por R. E. Fry, pp. 47-65. Paper 1 Society for American Archaeology, Washington D.C.

Bishop, R., R.L. Rands y G.R. Holley

1982 Ceramic compositional analysis in archaeological perspective. En Advances in Archaeological Method and Theory 7, editado por M. Schiffer, pp. 275-330. Academic Press, New York.

Bishop, R. y H. Neff

1989 Compositional data analysis in archaeology. En $\mathrm{Ar}$ chaeological Chemistry I. Advances in Chemistry, Series 220, editado por R. O. Allen, pp. 57-86. American Chemical Society, Washington, D. C.

Bouysse-Cassagne, $\mathrm{T}$.

1997 Introducción. En Saberes y Memorias en los Andes, editado y compilado por T. Bouysse-Cassagne, pp. 7-12. CREDAL-IFEA, Lima.

Bubba, C.

1997 Los rituales a los vestidos de María Titiqhawa, Juana Palla y otros fundadores de los ayllu de Coroma. En Memoria Ritual. Saberes y Memorias en los Andes, editado y compilado por T. Bouysse-Cassagne, pp. 377- 400. CREDAL-IFEA, Lima.

Candau, J.

2002 Antropología de la Memoria. Nueva Visión. Buenos Aires.

Connerton. P.

1989 How societies remember. En Social Memory, pp. 6-40. Cambridge University Press, Cambridge.

D'Altroy, T. y R. Bishop

1990 The provincial organization of inka ceramic production. American Antiquity 55(1):120-137.

De La Fuente, G.

2002 Aplicación de un Bioindicador Arqueológico (Diatomeas) para el Estudio de Fuentes de Aprovisionamiento de Arcillas. CENEDIT, Catamarca.

Desrosiers, $\mathrm{S}$.

1997 Lógicas textiles y lógicas culturales en los Andes. En Saberes y Memorias en los Andes, editado y compilado por T. Bouysse-Cassagne, pp. 325-349. CREDAL-IFEA, Lima.

Dollfus, O.

1991 Del reto del espacio andino a los Andes como lugares de memoria. En Territorios Andinos. Reto y memoria, pp. 15-28. IFEA-IEP, Lima.
Feely, A.

2003 Propiedades del registro y variabilidad tecno-morfológica cerámica: vía de análisis para acceder a la funcionalidad del sitio arqueológico de Batungasta (Dpto. Tinogasta, Catamarca). Tesis para optar al grado de Licenciado en Antropología, Mención Arqueología, Departamento de Ciencias Antropológicas, Facultad de Filosofía y Letras, Universidad de Buenos Aires, Buenos Aires.

Flores Ochoa, J.

1990 Gráfica inca y tradición oral. Oralidad. Anuario para el Rescate de la Tradición Oral de América Latina y el Caribe 2:53-58.

Harris, O.

1997 Los límites como problema: mapas etnohistóricos de los Andes bolivianos. En Saberes y Memorias en los Andes, editado y compilado por T. Bouysse-Cassagne, pp. 351373. CREDAL-IFEA, Lima.

Hastorf, C.

2003 Community with the ancestors: ceremonies and social memory in the Middle Formative at Chiripa, Bolivia. Journal of Anthropological Archaeology 22(4):305-332.

Lemonier, $\mathrm{P}$.

1992 Elements for an anthropology of technology. Anthropological Papers $\mathrm{N}^{\circ} 88$, University of Michigan. Ann Arbor, Michigan.

Neff, H., editor

1992 Chemical Characterization of Ceramic Pastes in Archaeology. Prehistory Press, Madison, Wiscosin.

Neff, $\mathrm{H}$.

1998 Units in Chemistry-Bases Ceramic Provenance Investigations. En Unit Issues in Archaeology, editado por A. Ramenofsky y A. Steffen, pp. 115-147. University of Utah Press, Salt Lake.

Plá, R. y N. Ratto

2003 Provenience archaeological studies of ceramic raw material and artifacts using instrumental neutron activation analysis: the cases of Chaschuil and Bolsón de Fiambalá (Catamarca, Argentina). En Nuclear Analytical Techniques in Archaeological Investigations. Report Series 416, pp. 7-22. International Atomic Energy Agency. Vienna.

Ratto, N.

$2000 \mathrm{La}$ estructura del registro arqueológico en la cuenca superior del valle de Chaschuil (Dpto. Tinogasta, Catamarca). Arqueología 10:39-78.

2003 Estrategias de Caza y Propiedades del Registro Arqueológico en la Puna de Chaschuil (Dpto. Tinogasta, Catamarca). Tesis para optar al grado de Doctor en Filosofía y Letras, Mención Arqueología, Departamento de Ciencias Antropológicas, Facultad de Filosofía y Letras, Universidad de Buenos Aires, Buenos Aires.

Ratto, N. y M. Orgaz

2000 La ocupación Inka en la puna meridional catamarqueña: el caso de la cuenca superior del valle de Chaschuil (Departamento Tinogasta, Catamarca, Argentina). Tawantinsuyu, en prensa.

Ratto, N., M. Orgaz y R. Plá

2002a Producción y distribución de bienes cerámicos durante la ocupación Inka entre la región puneña de Chas- 
chuil y el valle de Abaucán (Dpto. Tinogasta, Catamarca). Relaciones de la Sociedad Argentina de Antropología 27:271-301.

Ratto, N., M. Orgaz, G. de La Fuente y R. Plá

2002b Ocupación de pisos de altura y contexto de producción cerámica durante el Formativo: el caso de la región puneña de Chaschuil y su relación con el Bolsón de Fiambalá (Dpto. Tinogasta, Catamarca, Argentina). Estudios Atacameños 24:51-69.

Sallnow, P.

1989 Precious metals in the Anden moral economy. En Money and the Morality of Exchange, editado por I. J. Parry and M. Bloch, pp. 209-231. Cambridge University Press. Cambridge.

Sempé, M.C.

1976 Contribución a la Arqueología del valle de Abaucán. Tesis para optar al grado de Doctor en Ciencias Naturales, Mención Arqueología, Universidad. Nacional de La Plata, La Plata.
1977 Las culturas agroalfareras prehispánicas del valle de Abaucán (Tinogasta-Catamarca). Relaciones de la Sociedad Argentina de Antropología (NS) 11:55-68.

Sillar, W.

1994 Pottery's Role in the Reproduction of Andean Society. Doctoral Dissertation, Department of Anthropology, University of Cambridge, Cambridge.

Stark, M.

1999 Social dimensions of technical choice in Kalinga ceramic traditions. En Material Meaning. Critical Approaches to the Interpretation of Material Culture, editado por E. Chilton, pp. 24-43. The University of Utah Press, Salt Lake. Zuidema, T.

1989a Parentesco y culto a los antepasados en tres comunidades peruanas. Una relación de Hernández de Príncipe de 1622. En Reyes y Guerreros. Ensayos de Cultura Andina, pp. 133-134. Fomciencias, Lima.

1989 b Las tumbas en pozos profundos y el imperio inca. En Reyes y Guerreros. Ensayos de Cultura Andina, pp. 149150. Fomciencias, Lima. 